Gut and Liver, Vol. 10, No. 1, January 2016, pp. 117-125

\title{
Prevalence and Factors Associated with Nonalcoholic Fatty Liver Disease in a Nonobese Korean Population
}

\author{
Hyun Chin Cho \\ Division of Gastroenterology, Department of Medicine, Samsung Changwon Hospital, Sungkyunkwan University School of Medicine, \\ Changwon, Korea
}

Background/Aims: Nonalcoholic fatty liver disease (NAFLD) is an emerging problem in Asia, but little is known about the disease in the nonobese population. The aims of this study were to investigate the prevalence of NAFLD and the factors associated with it in a nonobese Korean population and to compare the clinical characteristics of nonobese and obese subjects with NAFLD. Methods: This cross-sectional study used data from 2,058 subjects who participated in a medical checkup program. Results: The prevalence of NAFLD was $12.4 \%(213 / 1,711)$ in the nonobese population. A higher body mass index (BMI), higher homeostasis model assessment of insulin resistance (HOMA-IR) values, higher alanine aminotransferase (ALT) levels, triglyceride concentrations $\geq 150 \mathrm{mg} / \mathrm{dL}$, and hyperuricemia were independently associated with the presence of NAFLD in the nonobese subjects. Compared with the obese subjects with NAFLD, the nonobese subjects with NAFLD were composed of a higher proportion of females and had lower BMls, smaller waist circumferences, lower HOMA-IR values, and fewer metabolic irregularities. Conclusions: Higher BMls, HOMA-IR values, ALT levels, hypertriglyceridemia, and hyperuricemia were associated with NAFLD in the nonobese subjects. Clinicians should be particularly aware of the possibility of NAFLD in nonobese Asian people. (Gut Liver 2016;10:117-125)

Key Words: Non-alcoholic fatty liver disease; Nonobese population

\section{INTRODUCTION}

Nonalcoholic fatty liver disease (NAFLD) is increasingly being identified within westernized populations and it is an emerging problem in Asia where subjects tend to be less obese than their westernized counterparts. ${ }^{1-5}$ NAFLD is a chronic liver disease that is seen in the absence of secondary causes for the accumulation of hepatic fat. It has a wide histological spectrum that ranges from simple hepatic steatosis to steatohepatitis, liver cirrhosis, and even hepatocellular carcinoma. ${ }^{3,6}$ A high prevalence of NAFLD, which ranges from $18 \%$ to $45 \%$, has been reported among various ethnic groups, and it is associated with the growing obesity epidemic. $^{7-9}$

Most of the epidemiological studies that have investigated NAFLD have been conducted in obese populations. Consequently, much less is known about NAFLD in nonobese people, although NAFLD is not uncommon in these populations. ${ }^{10,11}$ To improve the management of NAFLD and to prevent its development, it is important to gain an understanding of the prevalence of NAFLD in nonobese populations, understand the factors associated with NAFLD in nonobese populations, and to determine whether differences exist between nonobese and obese populations in relation to the factors that are associated with NAFLD.

The aims of this study were to investigate the prevalence of NAFLD and the factors associated with the presence of NAFLD in a nonobese Korean population, and to compare the clinical characteristics of nonobese and obese subjects with NAFLD.

\section{MATERIALS AND METHODS}

\section{Study population}

This cross-sectional study used data from individuals who participated in a medical checkup program that was undertaken at the Health Promotion Center of the Samsung Changwon

Correspondence to: Hyun Chin Cho

Division of Gastroenterology, Department of Medicine, Samsung Changwon Hospital, Sungkyunkwan University School of Medicine, 158 Paryongro, Masanhoewon-gu, Changwon 51353, Korea

Current Affiliation: Department of Internal Medicine and Institute of Health Sciences, Gyeongsang National University School of Medicine, 15 Jinju-daero 816beon-gil, Jinju 52727, Korea

Tel: +82-55-750-9490, Fax: +82-55-750-9496, E-mail: academic77@naver.com

Received on November 13, 2014. Revised on February 8, 2015. Accepted on February 23, 2015. Published online August 11, 2015 pISSN 1976-2283 eISSN 2005-1212 http://dx.doi.org/10.5009/gnl14444

@) This is an Open Access article distributed under the terms of the Creative Commons Attribution Non-Commercial License (http://creativecommons.org/licenses/by-nc/4.0) which permits unrestricted non-commercial use, distribution, and reproduction in any medium, provided the original work is properly cited. 
Hospital from January 2014 to April 2014. The medical checkup program involved 12,567 participants during this timeframe, and individuals were excluded from this study if they did not have data relating to abdominal ultrasonography findings, serum insulin levels, or the presence of positive serologic markers for the hepatitis B or hepatitis C viruses. Individuals were also excluded from the study if they consumed excessive amounts of alcohol (>210 g per week for men and >140 g per week for women), had autoimmune hepatitis, or if they had other known causes of chronic liver disease. Subjects who were taking hepatotoxic, antihypertensive, antidiabetic, lipid-lowering, or hypouricemic medications were also excluded from the study. In addition, subjects who did not have NAFLD and had a body mass index (BMI) of $\geq 25 \mathrm{~kg} / \mathrm{m}^{2}$ were excluded from the study. Consequently, 2,058 subjects who were aged over 18 years were included in this study. The Institutional Review Board of Samsung Changwon Hospital approved this retrospective study.

\section{Anthropometric and laboratory measurements}

A trained technician performed the anthropometric measurements. Body weight and height were measured to the nearest $0.1 \mathrm{~kg}$ and $0.1 \mathrm{~cm}$, respectively, with the subjects wearing light clothes and not wearing shoes. The BMI was calculated by dividing the weight in kilograms by the square of the height in meters. A single assessor measured the circumference of the waist at the level of the umbilicus while the subject was standing. Blood pressure was measured using a standard sphygmomanometer after the subjects had rested for 5 minutes.

The subjects' biochemical parameters were evaluated after they had fasted overnight. The biochemical parameters assessed included levels of total cholesterol, triglyceride, low-density lipoprotein (LDL) cholesterol, high-density lipoprotein (HDL) cholesterol, fasting blood glucose, uric acid, total bilirubin, aspartate aminotransferase (AST), alanine aminotransferase (ALT), $\gamma$-glutamyltransferase $(\gamma$-GTP), and C-reactive protein (CRP), which were measured using a Hitachi Modular D2400 automated analyzer (Roche, Tokyo, Japan). Fasting insulin concentrations were determined using a Hitachi Modular Analytics E170 electrochemiluminescence immunoassay (Roche). Platelet counts were evaluated using a Sysmex XE2100 hematology analyzer (Sysmex Corp., Kobe, Japan).

Insulin resistance was determined using the homeostasis model assessment of insulin resistance (HOMA-IR) and values were obtained using the following formula: HOMA-IR=fasting insulin (IU/mL) xfasting blood glucose $(\mathrm{mmol} / \mathrm{L}) / 22.5{ }^{12}$

\section{Assessment of the presence or absence of NAFLD}

Abdominal ultrasonography using a Logiq 7 3.5-MHz convextype transducer (GE Healthcare, Milwaukee, WI, USA) determined the presence or absence of NAFLD. The ultrasonography procedure was performed by one of 10 experienced radiologists at the Health Promotion Center. The images were captured while the patient was in the supine position with their right arm raised above their head. The ultrasonography images of mild fatty liver were reviewed by two experienced radiologists. Fatty liver was diagnosed using well-established criteria, including the hepatorenal echo contrast (false transonic aspect of the right kidney as compared to liver parenchyma), liver parenchymal brightness (due to the high number of highly reflective interfaces produced by increased intracellular fat deposition), deep beam attenuation, and vascular blurring (because the increased liver echogenicity leads to decreased acoustic impedance between the parenchyma and the portal venule walls). ${ }^{2,13}$ The first two were used as definitive criteria, and the last two were taken into account as needed.

\section{Definitions}

We defined obesity as a BMI of $\geq 25 \mathrm{~kg} / \mathrm{m}^{2}$ in accordance with the World Health Organization's criteria for Asian people. ${ }^{14}$

The metabolic syndrome was defined in accordance with the criteria for Asian American people proposed by the National Cholesterol Education Program (NCEP) Adult Treatment Panel (ATP) III. ${ }^{15}$ The panel specified that a diagnosis of the metabolic syndrome should be comprised of the presence of any three of the five components that follow: a waist circumference of $\geq 90$ $\mathrm{cm}$ for men or $\geq 80 \mathrm{~cm}$ for women; blood pressure of $\geq 130 / 85$ $\mathrm{mm} \mathrm{Hg}$ or the current use of antihypertensive drugs; triglyceride levels of $\geq 150 \mathrm{mg} / \mathrm{dL}$; HDL cholesterol levels of $<40 \mathrm{mg} / \mathrm{dL}$ for men or $<50 \mathrm{mg} / \mathrm{dL}$ for women; and fasting blood glucose levels of $\geq 100 \mathrm{mg} / \mathrm{dL}$ or the current use of drugs for hyperglycemia.

Abdominal obesity was defined according to the ATP III criteria, that is, a waist circumference of $\geq 90 \mathrm{~cm}$ for men or $\geq 80 \mathrm{~cm}$ for women.

Dyslipidemia was defined using the NCEP guidelines, ${ }^{16}$ as follows: a total cholesterol level of $\geq 200 \mathrm{mg} / \mathrm{dL}$; a triglyceride level of $\geq 150 \mathrm{mg} / \mathrm{dL}$; an LDL cholesterol level of $>100 \mathrm{mg} / \mathrm{dL}$; and an HDL cholesterol level of $<40 \mathrm{mg} / \mathrm{dL}$ for men or $<50 \mathrm{mg} / \mathrm{dL}$ for women.

Hyperuricemia was defined as a uric acid level of $\geq 7 \mathrm{mg} / \mathrm{dL}$ in men or $\geq 6.5 \mathrm{mg} / \mathrm{dL}$ in women. ${ }^{11}$

\section{Statistical analysis}

Continuous variables are expressed as the means ( \pm standard deviations) or the medians and the interquartile ranges, and they were compared using Student t-test or the Mann-Whitney $\mathrm{U}$ test, depending on the normality of the data. Categorical variables were compared using the chi-square test. For the continuous variables, effect sizes were determined using Cohen's $d$ and they were calculated using the difference between the means of the two groups and the pooled within-group standard deviation. Effect sizes were classified as small $(d=0.2)$, medium $(d=0.5)$, or large $(d \geq 0.8)$. For the categorical variables, effect sizes were determined using the odds ratios (OR) of the two groups, and effect sizes were classified as small (OR, 1.5), medium (OR, 2), or 
large (OR $\geq 3) .{ }^{17}$ To evaluate the factors associated with the presence of NAFLD, multivariate binary logistic regression analysis was performed using the variables that had p-values of $<0.05$ in the univariate analysis. Numerical variables that had standard cutoff values that were provided by the ATP III criteria or the World Health Organization, including waist circumference, blood pressure, fasting blood glucose levels, triglyceride levels, HDL cholesterol levels, and the BMI, were used as dichotomous variables.

A two-tailed p-value of $<0.05$ was considered statistically significant. All of the statistical analyses were conducted using IBM SPSS software version 21.0 (IBM, Armonk, NY, USA). Cohen's $d$ was calculated using the effect size calculator for a Student t-test that is available from http://www.danielsoper.com/ statcalc.

\section{RESULTS}

\section{Clinical characteristics of the nonobese subjects with and without NAFLD}

The clinical features of the nonobese subjects with and without NAFLD are presented in Table 1. The prevalence of NAFLD was $12.4 \%(213 / 1,711)$ in the nonobese population.

The nonobese subjects with NAFLD tended to be older, be male, have the metabolic syndrome, and have larger waist circumferences, and they had higher BMIs, systolic and diastolic blood pressures, HOMA-IR values, total cholesterol levels, triglyceride levels, LDL cholesterol levels, fasting blood glucose levels, serum uric acid levels, total bilirubin levels, AST levels, ALT levels, $\gamma$-GTP levels, platelet counts, and CRP levels. The individuals in the nonobese NAFLD group had lower HDL cholesterol levels than the nonobese subjects without NAFLD. Among these variables, being male, the BMI, the waist circumference, the triglyceride levels, the HDL cholesterol levels, the HOMAIR values, the uric acid levels, the ALT levels, and the metabolic syndrome showed larger than medium effect sizes.

\section{Clinical characteristics of the nonobese and obese sub- jects with NAFLD}

The clinical characteristics of the nonobese and obese subjects with NAFLD are shown in Table 2. There was a male predominance within the group of nonobese subjects with NAFLD, but compared with the obese subjects with NAFLD, this group was comprised of a relatively higher proportion of females. Com-

Table 1. Clinical Characteristics of the Nonobese Subjects with or without Nonalcoholic Fatty Liver Disease

\begin{tabular}{|c|c|c|c|c|}
\hline Variable & $\begin{array}{l}\text { Without NAFLD } \\
\qquad(\mathrm{n}=1,498)\end{array}$ & $\begin{array}{l}\text { With NAFLD } \\
\quad(n=213)\end{array}$ & $\mathrm{p}$-value & Effect size \\
\hline Age, yr & $43 \pm 6.5$ & $44 \pm 6.7$ & 0.019 & 0.15 \\
\hline Male sex, n (\%) & $1,182(78.9)$ & $191(89.7)$ & $<0.001$ & 2.32 \\
\hline Body mass index, $\mathrm{kg} / \mathrm{m}^{2}$ & $22.2(16.1-24.9)$ & 23.7 (19.9-24.9) & $<0.001$ & 1.08 \\
\hline Waist circumference, $\mathrm{cm}$ & $78.2 \pm 5$ & $83.5 \pm 4.1$ & $<0.001$ & 1.16 \\
\hline \multicolumn{5}{|l|}{ Blood pressure, $\mathrm{mm} \mathrm{Hg}$} \\
\hline Systolic & $118(90-138)$ & $123(92-138)$ & $<0.001$ & 0.38 \\
\hline Diastolic & $69(43-89)$ & $73(51-88)$ & $<0.001$ & 0.35 \\
\hline Total cholesterol, mg/dL & $189 \pm 31.7$ & $200.6 \pm 34.6$ & $<0.001$ & 0.35 \\
\hline Triglycerides, mg/dL & 77 (30-362) & $135.2(32-376)$ & $<0.001$ & 0.68 \\
\hline LDL cholesterol, mg/dL & $121.7 \pm 30.1$ & $134.9 \pm 33.1$ & $<0.001$ & 0.42 \\
\hline HDL cholesterol, mg/dL & $59 \pm 13.6$ & $51.1 \pm 10.5$ & $<0.001$ & 0.65 \\
\hline Fasting glucose, mg/dL & $85.2 \pm 8.3$ & $89 \pm 8.8$ & $<0.001$ & 0.44 \\
\hline HOMA-IR & $0.67(0.03-5.99)$ & $1.05(0.05-2.88)$ & $<0.001$ & 0.73 \\
\hline Uric acid, mg/dL & $5.1 \pm 1.2$ & $5.8 \pm 1.2$ & $<0.001$ & 0.58 \\
\hline Total bilirubin, mg/dL & $0.8 \pm 0.3$ & $0.9 \pm 0.4$ & 0.043 & 0.28 \\
\hline AST, IU/L & $22.1 \pm 7.8$ & $27.1 \pm 14.4$ & $<0.001$ & 0.43 \\
\hline ALT, IU/L & $18.6 \pm 11.4$ & $30.7 \pm 25.8$ & $<0.001$ & 0.61 \\
\hline$\gamma$-GTP, IU/L & $25.4 \pm 29$ & $40.4 \pm 32.6$ & $<0.001$ & 0.49 \\
\hline Platelet count, $\times 10^{3} / \mu \mathrm{L}$ & $239 \pm 50$ & $248 \pm 49$ & 0.018 & 0.18 \\
\hline $\mathrm{CRP}, \mathrm{mg} / \mathrm{L}$ & $0.3(0.2-68)$ & $0.5(0.2-19.4)$ & $<0.001$ & 0.06 \\
\hline Metabolic syndrome, n (\%) & $26(1.7)$ & $19(8.9)$ & $<0.001$ & 5.55 \\
\hline
\end{tabular}

Data are presented as mean \pm SD or median (interquartile range) unless otherwise indicated. Effect sizes are expressed as Cohen’s $d$ and odds ratios. NAFLD, nonalcoholic fatty liver disease; LDL, low-density lipoprotein; HDL, high-density lipoprotein; HOMA-IR, homeostasis model assessment of insulin resistance; AST, aspartate aminotransferase; ALT, alanine aminotransferase; $\gamma$-GTP, $\gamma$-glutamyltransferase; CRP, C-reactive protein. 
Table 2. Clinical Characteristics of the Nonobese and Obese Nonalcoholic Fatty Liver Disease Subjects

\begin{tabular}{|c|c|c|c|c|}
\hline Variable & $\begin{array}{c}\text { Nonobese } \\
(n=213)\end{array}$ & $\begin{array}{c}\text { Obese } \\
(n=347)\end{array}$ & $\mathrm{p}$-value & Effect size \\
\hline Age, yr & $44 \pm 6.7$ & $43.5 \pm 7.4$ & 0.278 & 0.01 \\
\hline Male sex, n (\%) & $191(89.7)$ & $335(96.5)$ & 0.001 & 3.22 \\
\hline Body mass index, $\mathrm{kg} / \mathrm{m}^{2}$ & 23.7 (19.9-24.9) & $26.9(25-40.5)$ & $<0.001$ & 2.38 \\
\hline Waist circumference, cm & $83.5 \pm 4.1$ & $91.2 \pm 5$ & $<0.001$ & 1.68 \\
\hline \multicolumn{5}{|l|}{ Blood pressure, mm Hg } \\
\hline Systolic & $123(92-138)$ & $125(90-139)$ & 0.026 & 0.20 \\
\hline Diastolic & $73(51-88)$ & $75(47-88)$ & 0.006 & 0.22 \\
\hline Total cholesterol, mg/dL & $200.6 \pm 34.6$ & $199.6 \pm 34$ & 0.982 & 0.03 \\
\hline Triglycerides, mg/dL & $135.2(32-376)$ & $127(26-532)$ & 0.046 & 0.16 \\
\hline LDL cholesterol, mg/dL & $134.9 \pm 33.1$ & $134.8 \pm 31.7$ & 0.710 & 0.01 \\
\hline HDL cholesterol, mg/dL & $51.1 \pm 10.5$ & $47.4 \pm 10.2$ & $<0.001$ & 0.36 \\
\hline Fasting glucose, mg/dL & $89 \pm 8.8$ & $90.3 \pm 8.7$ & 0.045 & 0.15 \\
\hline HOMA-IR & $1.05(0.05-2.88)$ & $1.35(0.25-5.96)$ & $<0.001$ & 0.60 \\
\hline Uric acid, mg/dL & $5.8 \pm 1.2$ & $6 \pm 1.2$ & 0.036 & 0.18 \\
\hline Total bilirubin, mg/dL & $0.9 \pm 0.4$ & $0.9 \pm 0.4$ & 0.874 & 0.03 \\
\hline AST, IU/L & $27.1 \pm 14.4$ & $27.7 \pm 11.8$ & 0.081 & 0.05 \\
\hline ALT, IU/L & $30.7 \pm 25.8$ & $34.3 \pm 20.8$ & 0.001 & 0.15 \\
\hline$\gamma$-GTP, IU/L & $40.4 \pm 32.6$ & $44.5 \pm 33.7$ & 0.004 & 0.12 \\
\hline Platelet count, $\times 10^{3} / \mu \mathrm{L}$ & $248 \pm 49$ & $241.5 \pm 51.2$ & 0.087 & 0.12 \\
\hline $\mathrm{CRP}, \mathrm{mg} / \mathrm{L}$ & $0.5(0.2-19.4)$ & $0.70(0.15-101.26)$ & $<0.001$ & 0.12 \\
\hline Metabolic syndrome, n (\%) & $19(8.9)$ & $63(18.2)$ & 0.003 & 2.27 \\
\hline
\end{tabular}

Data are presented as mean \pm SD or median (interquartile range) unless otherwise indicated. Effect sizes are expressed as Cohen’s $d$ and odds ratios. LDL, low-density lipoprotein; HDL, high-density lipoprotein; HOMA-IR, homeostasis model assessment of insulin resistance; AST, aspartate aminotransferase; ALT, alanine aminotransferase; $\gamma$-GTP, $\gamma$-glutamyltransferase; CRP, C-reactive protein.

pared with the obese subjects with NAFLD, the nonobese subjects with NAFLD had lower BMIs, smaller waist circumferences, lower systolic and diastolic blood pressures, lower HOMA-IR values, a lower prevalence of the metabolic syndrome, lower triglyceride levels, lower fasting blood glucose levels, lower serum uric acid levels, lower ALT levels, lower $\gamma$-GTP levels, and lower CRP levels. The nonobese subjects with NAFLD had higher levels of HDL cholesterol compared with obese subjects with NAFLD. Among these variables, sex, the BMI, the waist circumference, the HOMA-IR value, and the metabolic syndrome showed larger than medium effect sizes.

\section{Factors associated with the presence of NAFLD in the nonobese subjects}

Univariate binary logistic regression analysis identified the following as significant factors associated with the presence of NAFLD in the nonobese subjects (Table 3): being older, being male, abdominal obesity, a systolic blood pressure of $\geq 130 \mathrm{~mm}$ $\mathrm{Hg}$, a total cholesterol level of $\geq 200 \mathrm{mg} / \mathrm{dL}$, a triglyceride level of $\geq 150 \mathrm{mg} / \mathrm{dL}$, an LDL cholesterol level of $>100 \mathrm{mg} / \mathrm{dL}$, an HDL cholesterol level of $<40 \mathrm{mg} / \mathrm{dL}$ for men or $<50 \mathrm{mg} / \mathrm{dL}$ for wom- en, a fasting blood glucose level of $\geq 100 \mathrm{mg} / \mathrm{dL}$, hyperuricemia, the presence of the metabolic syndrome, a higher BMI, higher HOMA-IR values, and higher levels of total bilirubin, AST, ALT, $\gamma$-GTP, and platelets.

Multivariate binary logistic regression analysis determined that higher BMIs $(\mathrm{p}<0.001)$, higher HOMA-IR values $(\mathrm{p}<0.001)$, higher ALT levels $(\mathrm{p}<0.001)$, triglyceride levels of $\geq 150 \mathrm{mg} / \mathrm{dL}$ $(\mathrm{p}=0.021)$, and hyperuricemia $(\mathrm{p}=0.001)$ were significant and independent factors associated with the presence of NAFLD in the nonobese subjects (Table 4).

To explore the relationships between NAFLD and the HOMAIR values or the serum uric acid levels, the HOMA-IR values and the serum uric acid levels were grouped into quartiles. Multivariate binary logistic regression analysis showed that the HOMAIR $(\mathrm{p}<0.001)$ and serum uric acid $(\mathrm{p}=0.007)$ quartiles remained significant and independent factors associated with the presence of NAFLD in the nonobese subjects. Compared with the HOMAIR quartile (Q) 1 values, the OR for the HOMA-IR Q4 values (1.04 to 5.99) was 3.190 (95\% confidence interval [CI], 1.818 to 5.598; $\mathrm{p}<0.001$ ) (Fig. 1). Compared with the serum uric acid Q1 values, the OR for the serum uric acid Q4 values (6.1 to $8.9 \mathrm{mg} / \mathrm{dL}$ ) was 
Table 3. Factors Associated with the Presence of Nonalcoholic Fatty Liver Disease in the Nonobese Subjects Evaluated Using Univariate Binary Logistic Regression Analysis

\begin{tabular}{|c|c|c|c|c|}
\hline Variable & NAFLD & p-value & OR & $95 \% \mathrm{CI}$ \\
\hline Age, yr & $43 \pm 6.6$ & 0.019 & 1.026 & $1.004-1.049$ \\
\hline Male sex, n (\%) & $1,373(80.2)$ & $<0.001$ & 2.321 & $1.468-3.671$ \\
\hline Body mass index, $\mathrm{kg} / \mathrm{m}^{2}$ & $22.5(16.1-24.9)$ & $<0.001$ & 2.117 & $1.851-2.421$ \\
\hline Abdominal obesity, $\mathrm{n}(\%)^{*}$ & $80(4.7)$ & $<0.001$ & 2.860 & $1.711-4.779$ \\
\hline \multicolumn{5}{|l|}{ Blood pressure } \\
\hline Systolic $\geq 130$ mm Hg, n (\%) & $207(12.1)$ & 0.003 & 1.786 & $1.218-2.619$ \\
\hline Diastolic $\geq 85$ mm Hg, n (\%) & $78(4.6)$ & 0.423 & 1.295 & $0.688-2.438$ \\
\hline Total cholesterol $\geq 200 \mathrm{mg} / \mathrm{dL}, \mathrm{n}(\%)$ & $594(34.7)$ & $<0.001$ & 2.006 & $1.502-2.680$ \\
\hline Triglycerides $\geq 150 \mathrm{mg} / \mathrm{dL}, \mathrm{n}(\%)$ & $222(13)$ & $<0.001$ & 4.367 & $3.134-6.084$ \\
\hline LDL cholesterol >100 mg/dL, n (\%) & $1,300(76)$ & $<0.001$ & 2.278 & $1.505-3.446$ \\
\hline Reduced HDL cholesterol, n (\%)* & $146(8.5)$ & 0.002 & 1.953 & $1.270-3.003$ \\
\hline $\mathrm{FBG} \geq 100 \mathrm{mg} / \mathrm{dL}, \mathrm{n}(\%)$ & $102(6)$ & 0.010 & 1.939 & $1.172-3.208$ \\
\hline HOMA-IR & $0.7(0.03-5.99)$ & $<0.001$ & 3.502 & $2.667-4.599$ \\
\hline Hyperuricemia, n $(\%)^{\dagger}$ & $140(8.2)$ & $<0.001$ & 3.509 & $2.364-5.207$ \\
\hline Total bilirubin, mg/dL & $0.8 \pm 0.4$ & 0.044 & 1.473 & $1.010-2.148$ \\
\hline AST, IU/L & $22.7 \pm 9.1$ & $<0.001$ & 1.046 & $1.031-1.061$ \\
\hline ALT, IU/L & $20.1 \pm 14.6$ & $<0.001$ & 1.050 & $1.039-1.061$ \\
\hline$\gamma$-GTP, IU/L & $27.3 \pm 29.9$ & $<0.001$ & 1.011 & $1.007-1.015$ \\
\hline Platelet count, $\times 10^{3} / \mu \mathrm{L}$ & $240 \pm 50$ & 0.019 & 1.003 & $1.001-1.006$ \\
\hline $\mathrm{CRP}, \mathrm{mg} / \mathrm{L}$ & $0.3(0.2-68)$ & 0.485 & 1.014 & $0.976-1.053$ \\
\hline Metabolic syndrome, n (\%) & $45(2.6)$ & $<0.001$ & 5.545 & $3.012-10.207$ \\
\hline
\end{tabular}

Data are presented as mean \pm SD or median (interquartile range) unless otherwise indicated.

NAFLD, nonalcoholic fatty liver disease; OR, odds ratio; CI, confidence interval; LDL, low-density lipoprotein; HDL, high-density lipoprotein; FBG, fasting blood glucose; HOMA-IR, homeostasis model assessment of insulin resistance; AST, aspartate aminotransferase; ALT, alanine aminotransferase; $\gamma$-GTP, $\gamma$-glutamyltransferase; CRP, C-reactive protein.

${ }^{*}$ According to the Adult Treatment Panel III criteria; ${ }^{\dagger}$ Hyperuricemia was defined as a uric acid level $\geq 7 \mathrm{mg} / \mathrm{dL}$ in men or $\geq 6.5 \mathrm{mg} / \mathrm{dL}$ in women.

2.506 (95\% CI, 1.405 to $4.473 ; \mathrm{p}=0.002$ ) (Fig. 2).

\section{DISCUSSION}

In the current study, the prevalence of NAFLD detected using ultrasound was $12.4 \%(213 / 1,711)$ in the nonobese (BMI $<25 \mathrm{~kg} / \mathrm{m}^{2}$ ) and nondiabetic population. Compared with the nonobese subjects without NAFLD, the nonobese subjects with NAFLD were older, predominantly male, and had higher BMIs, larger waist circumferences, higher systolic and diastolic blood pressures, higher HOMA-IR values, and an increased prevalence of the metabolic syndrome. In addition, these individuals had higher total cholesterol levels, triglyceride levels, LDL cholesterol levels, fasting blood glucose levels, serum uric acid levels, total bilirubin levels, AST levels, ALT levels, $\gamma$-GTP levels, platelet counts, and CRP levels, and lower HDL cholesterol levels, compared with the nonobese subjects without NAFLD. Higher BMIs, higher HOMA-IR values, higher ALT levels, triglyceride levels of $\geq 150 \mathrm{mg} / \mathrm{dL}$, and hyperuricemia were significant and independent factors associated with the presence of NAFLD in the nonobese subjects. Compared with the obese subjects with NAFLD, the nonobese subjects with NAFLD were comprised of a relatively higher proportion of females, and they had higher HDL cholesterol levels, lower BMIs, smaller waist circumferences, lower systolic and diastolic blood pressures, lower HOMA-IR values, a lower prevalence of the metabolic syndrome, lower triglyceride levels, lower fasting blood glucose levels, lower serum uric acid levels, lower ALT levels, lower $\gamma$-GTP levels, and lower CRP levels.

The prevalence of NAFLD has increased rapidly over the past two decades in the Asia-Pacific region. NAFLD is not uncommon in subjects who are considered nonobese. Previous studies have reported a prevalence of NAFLD that ranges from 7.27\% to $23.4 \%$ in the nonobese population. ${ }^{11,18,19}$ In the current study, the prevalence of NAFLD was $12.4 \%$ in the nonobese, nondiabetic population.

The current study demonstrates that the nonobese subjects with NAFLD were predominantly male, but compared with the obese subjects with NAFLD, they were comprised of a relatively higher proportion of females, and they had lower BMIs, smaller 
Table 4. Factors Associated with the Presence of Nonalcoholic Fatty Liver Disease in the Nonobese Subjects Evaluated Using Multivariate Binary Logistic Regression Analysis

\begin{tabular}{|c|c|c|c|c|}
\hline Variable & NAFLD & p-value & $\mathrm{OR}$ & $95 \% \mathrm{CI}$ \\
\hline Age, yr & $43 \pm 6.6$ & 1.109 & 1.021 & $0.995-1.047$ \\
\hline Male sex, n (\%) & $1,373(80.2)$ & 0.662 & 1.179 & $0.564-2.462$ \\
\hline Body mass index, $\mathrm{kg} / \mathrm{m}^{2}$ & $22.5(16.1-24.9)$ & $<0.001$ & 1.925 & $1.651-2.244$ \\
\hline Abdominal obesity, $\mathrm{n}(\%)^{*}$ & $80(4.7)$ & 0.165 & 1.836 & $0.779-4.332$ \\
\hline $\mathrm{SBP} \geq 130 \mathrm{~mm} \mathrm{Hg}, \mathrm{n}(\%)$ & $207(12.1)$ & 0.783 & 0.933 & $0.571-1.526$ \\
\hline Total cholesterol $\geq 200 \mathrm{mg} / \mathrm{dL}, \mathrm{n}(\%)$ & $594(34.7)$ & 0.544 & 1.121 & $0.775-1.623$ \\
\hline Triglycerides $\geq 150 \mathrm{mg} / \mathrm{dL}, \mathrm{n}(\%)$ & $222(13)$ & 0.021 & 1.701 & $1.085-2.666$ \\
\hline LDL cholesterol >100 mg/dL, n (\%) & $1,300(76)$ & 0.139 & 1.462 & $0.884-2.416$ \\
\hline Reduced HDL cholesterol, $n(\%)^{*}$ & $146(8.5)$ & 0.200 & 0.669 & $0.361-1.237$ \\
\hline $\mathrm{FBG} \geq 100 \mathrm{mg} / \mathrm{dL}, \mathrm{n}(\%)$ & $102(6)$ & 0.619 & 0.843 & $0.431-1.650$ \\
\hline HOMA-IR & $0.7(0.03-5.99)$ & $<0.001$ & 2.498 & $1.821-3.428$ \\
\hline Hyperuricemia, $n(\%)^{\dagger}$ & $140(8.2)$ & 0.001 & 2.200 & $1.365-3.546$ \\
\hline Total bilirubin, mg/dL & $0.8 \pm 0.4$ & 0.120 & 1.432 & $0.911-2.251$ \\
\hline AST, IU/L & $22.7 \pm 9.1$ & 0.941 & 0.999 & $0.975-1.024$ \\
\hline ALT, IU/L & $20.1 \pm 14.6$ & $<0.001$ & 1.038 & $1.019-1.056$ \\
\hline$\gamma$-GTP, IU/L & $27.3 \pm 29.9$ & 0.745 & 0.999 & $0.994-1.005$ \\
\hline Platelet count, $\times 10^{3} / \mu \mathrm{L}$ & $240 \pm 50$ & 0.087 & 1.003 & $1.000-1.006$ \\
\hline Metabolic syndrome, $\mathrm{n}(\%)$ & $45(2.6)$ & 0.149 & 2.030 & $0.776-5.307$ \\
\hline
\end{tabular}

Data are presented as mean \pm SD or median (interquartile range) unless otherwise indicated.

NAFLD, nonalcoholic fatty liver disease; OR, odds ratio; CI, confidence interval; SBP, systolic blood pressure; LDL, low-density lipoprotein; HDL, high-density lipoprotein; FBG, fasting blood glucose; HOMA-IR, homeostasis model assessment of insulin resistance; AST, aspartate aminotransferase; ALT, alanine aminotransferase; $\gamma$-GTP, $\gamma$-glutamyltransferase.

${ }^{*}$ According to the Adult Treatment Panel III criteria; ${ }^{\dagger}$ Hyperuricemia was defined as a uric acid level $\geq 7 \mathrm{mg} / \mathrm{dL}$ in men or $\geq 6.5 \mathrm{mg} / \mathrm{dL}$ in women.

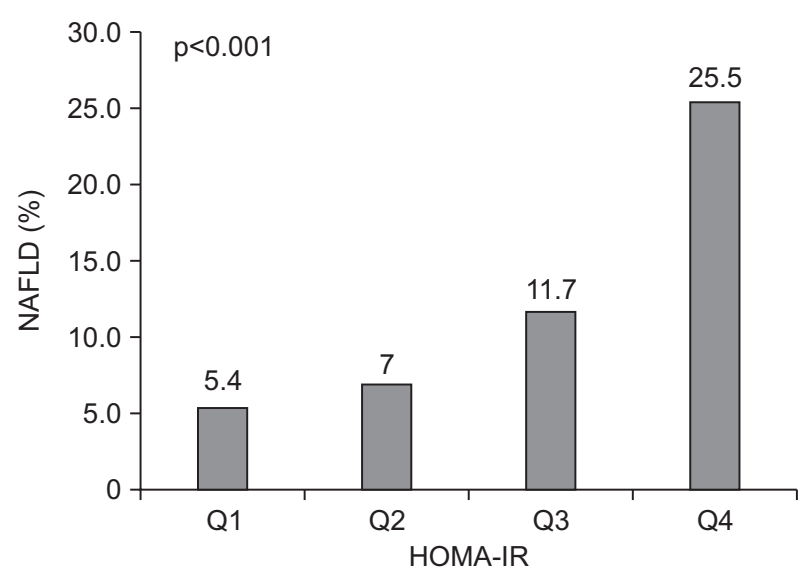

Fig. 1. The prevalence of nonalcoholic fatty liver disease (NAFLD) according to the homeostasis model assessment of insulin resistance (HOMA-IR) quartiles. Multivariate analysis using the variables described in Table 4.

Q1 (0.03-0.46): reference; Q2 (0.47-0.69): $\mathrm{p}=0.911$, odds ratio (OR), 0.966, 95\% confidence interval (CI), 0.525-1.775; Q3 (0.70-1.03): $\mathrm{p}=0.070, \mathrm{OR}, 1.696,95 \% \mathrm{CI}, 0.957-3.004$; Q4 (1.04-5.99): $\mathrm{p}<0.001$, OR, 3.190, 95\% CI, 1.818-5.598.

waist circumferences, lower insulin resistance levels, and fewer metabolic irregularities. Previous studies that have described NAFLD in nonobese subjects have also found differences in their

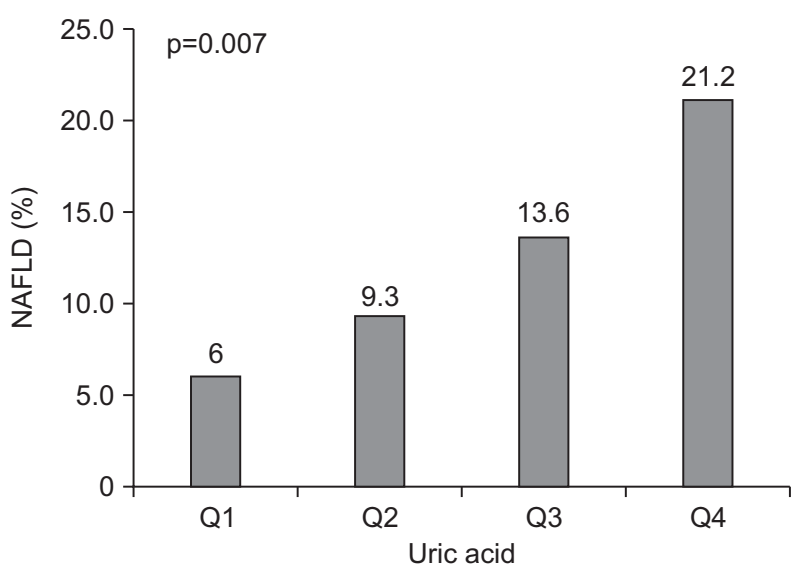

Fig. 2. The prevalence of nonalcoholic fatty liver disease (NAFLD) according to serum uric acid quartiles. Multivariate analysis using the variables described in Table 4.

Q1 (0.5-4.3 mg/dL): reference; Q2 (4.4-5.2 mg/dL): $p=0.302$, odds ratio (OR), 1.365, 95\% confidence interval (CI), 0.756-2.466; Q3 (5.3-6.0 $\mathrm{mg} / \mathrm{dL}$ ): $\mathrm{p}=0.065$, OR, 1.747, 95\% CI, 0.966-3.160; Q4 (6.1-8.9 mg/ $\mathrm{dL}): \mathrm{p}=0.002, \mathrm{OR}, 2.506,95 \% \mathrm{CI}, 1.405-4.473$.

clinical profiles compared with obese subjects with NAFLD. ${ }^{10,20,21}$

The relationship between obesity and NAFLD is well established. In the current study, BMI was a factor that was associat- 
ed with the presence of NAFLD, even in the nonobese subjects. $\mathrm{Xu}$ et $a{ }^{18}{ }^{18}$ reported that BMI was associated with the presence and development of NAFLD in nonobese subjects, while Kim et al. ${ }^{19}$ reported that body weight change was an important contributor to the development and regression of NAFLD in nonobese subjects. Thus, weight control to reduce the risk of NAFLD is important not only in obese individuals, but also in nonobese subjects.

Dyslipidemia is a well-known risk factor for NAFLD. In the current study, hypertriglyceridemia was a factor associated with the presence of NAFLD in the nonobese subjects. Several other studies have reported that hypertriglyceridemia is an important factor that is associated with the presence, development, and regression of NAFLD in nonobese subjects. ${ }^{10,11,18,19,22}$ It is generally considered that excessively high hepatic triglyceride concentrations and hypertriglyceridemia result from an increased influx of free fatty acids from visceral fat deposits and an increase in the secretion of very-low-density lipoprotein from the liver as a consequence of insulin resistance. ${ }^{23}$ However, recent findings from the Framingham Heart Study have suggested that fatty liver is associated with dyslipidemia and dysglycemia and is independent of fat deposits, including visceral adipose tissue. ${ }^{24}$ Although it is difficult to speculate about a pathogenic role for hypertriglyceridemia itself in the development of NAFLD based on the current evidence, serum triglyceride levels could be one of the markers for the progression of NAFLD, especially in nonobese subjects.

Insulin resistance provides an important pathological link to NAFLD and, therefore, the close association of NAFLD with metabolic irregularities. ${ }^{10,20,21,25}$ Sinn et al. ${ }^{25}$ reported that NAFLD is an independent predictor of insulin resistance, irrespective of the number of the metabolic components that comprise the metabolic syndrome that are present in nonobese Asian adults. The results of the current study suggest that insulin resistance plays a significant role in the pathogenesis of NAFLD in nonobese, nondiabetic subjects, irrespective of the presence of the metabolic syndrome. When the HOMA-IR values were grouped into quartiles and were analyzed using multivariate binary logistic regression analysis, the OR of the highest HOMA-IR quartile (1.04 to 5.99) was $3.190(\mathrm{p}<0.001)$, and the highest HOMA-IR quartile was associated with the highest prevalence of NAFLD (25.5\%). The correlation between NAFLD and insulin resistance was stronger compared with the correlation between NAFLD and the metabolic syndrome, and the current ATP III diagnostic criteria for the metabolic syndrome cannot identify NAFLD in nonobese subjects satisfactorily.

Several studies have demonstrated the association between serum uric acid and NAFLD in the general population, and even serum uric acid levels within the normal range are independently associated with the presence of NAFLD. ${ }^{26-29}$ A population-based prospective study of 6,890 initially NAFLD-free subjects showed that 813 subjects (11.8\%) developed NAFLD over a 3-year follow-up period, and that an elevation of serum uric acid levels independently predicted an increased risk of NAFLD. ${ }^{28}$ A cross-sectional study of 8,925 subjects (6,008 men) with a mean age of 43 years showed that NAFLD patients had significantly higher serum uric acid levels compared with the control group, and that an elevated serum uric acid level was an independent risk factor for NAFLD. ${ }^{26}$ Hyperuricemia is reportedly associated with insulin resistance and the metabolic syndrome. $^{30,31}$ The biochemical role of uric acid in NAFLD is poorly understood, and current data on this subject seem to be partially conflicting at best. The role played by increases in systemic oxidative stress in the progression of NAFLD has long been recognized in both animal experiments and clinical studies, ${ }^{32}$ and it has been proposed that uric acid acts as an antioxidant of peroxynitrite and peroxynitrite-derived radicals. ${ }^{33,34}$ A recent study showed that administering uric acid to obese (ob/ob) mice almost completely resolved fatty liver in these animals. ${ }^{35}$ Thus, elevated serum uric acid levels may reflect an underlying compensatory mechanism that counteracts the increased oxidative stress associated with NAFLD. On the contrary, uric acid becomes a strong oxidant in the context of the metabolic syndrome. ${ }^{36}$ Indeed, some investigators have proposed using allopurinol $^{37}$ or dietary measures, such as reducing fructose intakes, which is associated with increased uric acid levels, ${ }^{38}$ to treat the metabolic syndrome and NAFLD. Notably, hyperuricemia was found to be an important factor associated with NAFLD in the nonobese, nondiabetic subjects in the current study. When the levels of serum uric acid were grouped into quartiles and were analyzed using multivariate binary logistic regression analysis, the OR of the highest serum uric acid quartile (6.1 to $8.9 \mathrm{mg} / \mathrm{dL}$ ) was 2.506 ( $\mathrm{p}=0.002)$, and the highest serum uric acid quartile was associated with the highest prevalence of NAFLD (21.2\%). While the serum uric acid level was significantly associated with NAFLD in the nonobese subjects, future studies are needed to explain the physiological mechanism underlying this association.

In the current study, ALT was associated with the presence of NAFLD in the nonobese subjects. Prior study has suggested that elevated ALT levels correlate with the progression of NAFLD to steatohepatitis and hepatic fibrosis. ${ }^{39}$ But it is difficult to determine a systematic association between ALT level and NAFLD. Although elevated ALT levels merit attention in relation to the possible presence of NAFLD, an over-reliance on ALT levels underestimates the relevance of NAFLD. ${ }^{40}$ Further investigations are needed to explore the precise role of ALT level in nonobese subjects with NAFLD.

Although serum ferritin was not available in the current study, there have been several cross-sectional studies that showed that an elevated serum ferritin levels associated with insulin resistance and NALFD. ${ }^{41,42}$ Iron may potentially act as a source of oxidative stress and injury within in the liver. ${ }^{43}$ In animal models of fatty liver, iron loading is associated with the 
development of hepatic necro-inflammation and fibrosis. ${ }^{44}$ Cellbased and animal studies have demonstrated iron induces insulin resistance in adiposite tissue, the pancreas and liver. ${ }^{45,46}$

Recent study reported an association between serum irisin level and NAFLD. ${ }^{47}$ Irisin is a recently found myokine that aids obesity control and improves glucose hemostasis by acting on white adipose tissue cells and increases total energy consumption. Further investigations are needed to determine the role of serum irisin level in nonobese subjects with NAFLD.

There are several limitations to this study. First, this study was cross-sectional in its design; therefore, the natural course of NAFLD and causal relationships could not be determined. Second, the diagnosis of NAFLD was based on ultrasonography, which cannot detect mild steatosis. However, ultrasonography is widely used in epidemiological surveys of NAFLD, because it is noninvasive, safe, widely available, portable, and it has an acceptable sensitivity in relation to the detection of hepatic steatosis. Third, it is possible that a selection bias existed because the subjects who participated in the general medical checkup program were more concerned about their health compared with those who did not participate in the program. Furthermore, those individuals who did not have data relating to serum insulin levels were excluded from the current study, which might have affected the prevalence of NAFLD.

In conclusion, the prevalence of ultrasound-detected NAFLD was $12.4 \%(213 / 1,711)$ in the nonobese, nondiabetic population. Higher BMIs, higher HOMA-IR values, higher ALT levels, hypertriglyceridemia, and hyperuricemia were significant and independent factors associated with the presence of NAFLD in the nonobese subjects. Compared with the obese subjects with NAFLD, the nonobese subjects with NAFLD had a male predominance and they were comprised of a relatively higher proportion of females, and they had lower BMIs, smaller waist circumferences, lower levels of insulin resistance, and fewer metabolic irregularities. Hence, people in Asia are likely to develop NAFLD even in the absence of obesity. To improve the management of NAFLD and to prevent its development, clinicians should be particularly aware of the possibility of NAFLD in nonobese Asian people.

\section{CONFLICTS OF INTEREST}

No potential conflict of interest relevant to this article was reported.

\section{REFERENCES}

1. Angulo P. Nonalcoholic fatty liver disease. N Engl J Med 2002; 346:1221-1231.

2. Kojima S, Watanabe N, Numata M, Ogawa T, Matsuzaki S. Increase in the prevalence of fatty liver in Japan over the past 12 years: analysis of clinical background. J Gastroenterol 2003;38:
954-961.

3. Neuschwander-Tetri BA, Caldwell SH. Nonalcoholic steatohepatitis: summary of an AASLD Single Topic Conference. Hepatology 2003;37:1202-1219.

4. Farrell GC, Chitturi S, Lau GK, Sollano JD; Asia-Pacific Working Party on NAFLD. Guidelines for the assessment and management of non-alcoholic fatty liver disease in the Asia-Pacific region: executive summary. J Gastroenterol Hepatol 2007;22:775-777.

5. Das K, Das K, Mukherjee PS, et al. Nonobese population in a developing country has a high prevalence of nonalcoholic fatty liver and significant liver disease. Hepatology 2010;51:1593-1602.

6. Bellentani S, Scaglioni F, Marino M, Bedogni G. Epidemiology of non-alcoholic fatty liver disease. Dig Dis 2010;28:155-161.

7. Browning JD, Szczepaniak LS, Dobbins R, et al. Prevalence of hepatic steatosis in an urban population in the United States: impact of ethnicity. Hepatology 2004;40:1387-1395.

8. Bedogni G, Miglioli L, Masutti F, Tiribelli C, Marchesini G, Bellentani S. Prevalence of and risk factors for nonalcoholic fatty liver disease: the Dionysos nutrition and liver study. Hepatology 2005; 42:44-52.

9. Park SH, Jeon WK, Kim SH, et al. Prevalence and risk factors of non-alcoholic fatty liver disease among Korean adults. J Gastroenterol Hepatol 2006;21:138-143.

10. Kim HJ, Kim HJ, Lee KE, et al. Metabolic significance of nonalcoholic fatty liver disease in nonobese, nondiabetic adults. Arch Intern Med 2004;164:2169-2175.

11. Chen $\mathrm{CH}$, Huang $\mathrm{MH}$, Yang JC, et al. Prevalence and risk factors of nonalcoholic fatty liver disease in an adult population of Taiwan: metabolic significance of nonalcoholic fatty liver disease in nonobese adults. J Clin Gastroenterol 2006;40:745-752.

12. Matthews DR, Hosker JP, Rudenski AS, Naylor BA, Treacher DF, Turner RC. Homeostasis model assessment: insulin resistance and beta-cell function from fasting plasma glucose and insulin concentrations in man. Diabetologia 1985;28:412-419.

13. Needleman L, Kurtz AB, Rifkin MD, Cooper HS, Pasto ME, Goldberg BB. Sonography of diffuse benign liver disease: accuracy of pattern recognition and grading. AJR Am J Roentgenol 1986;146: 1011-1015.

14. WHO Expert Consultation. Appropriate body-mass index for Asian populations and its implications for policy and intervention strategies. Lancet 2004;363:157-163.

15. Grundy SM, Cleeman JI, Daniels SR, et al. Diagnosis and management of the metabolic syndrome: an American Heart Association/ National Heart, Lung, and Blood Institute Scientific Statement. Circulation 2005;112:2735-2752.

16. Expert Panel on Detection, Evaluation, and Treatment of High Blood Cholesterol in Adults. Executive Summary of the Third Report of the National Cholesterol Education Program (NCEP) Expert Panel on Detection, Evaluation, and Treatment of High Blood Cholesterol in Adults (Adult Treatment Panel III). JAMA 2001;285: 2486-2497.

17. Sullivan GM, Feinn R. Using effect size-or why the p value is not 
enough. J Grad Med Educ 2012;4:279-282.

18. Xu C, Yu C, Ma H, Xu L, Miao M, Li Y. Prevalence and risk factors for the development of nonalcoholic fatty liver disease in a nonobese Chinese population: the Zhejiang Zhenhai Study. Am J Gastroenterol 2013;108:1299-1304.

19. Kim NH, Kim JH, Kim YJ, et al. Clinical and metabolic factors associated with development and regression of nonalcoholic fatty liver disease in nonobese subjects. Liver Int 2014;34:604-611.

20. Bhat G, Baba CS, Pandey A, Kumari N, Choudhuri G. Insulin resistance and metabolic syndrome in nonobese Indian patients with non-alcoholic fatty liver disease. Trop Gastroenterol 2013;34:1824.

21. Vendhan R, Amutha A, Anjana RM, Unnikrishnan R, Deepa M, Mohan V. Comparison of characteristics between nonobese and overweight/obese subjects with nonalcoholic fatty liver disease in a South Indian population. Diabetes Technol Ther 2014;16:48-55.

22. Tsuneto A, Hida A, Sera N, et al. Fatty liver incidence and predictive variables. Hypertens Res 2010;33:638-643.

23. Cusi K. Role of obesity and lipotoxicity in the development of nonalcoholic steatohepatitis: pathophysiology and clinical implications. Gastroenterology 2012;142:711-725.

24. Speliotes EK, Massaro JM, Hoffmann U, et al. Fatty liver is associated with dyslipidemia and dysglycemia independent of visceral fat: the Framingham Heart Study. Hepatology 2010;51:19791987.

25. Sinn DH, Gwak GY, Park HN, et al. Ultrasonographically detected non-alcoholic fatty liver disease is an independent predictor for identifying patients with insulin resistance in non-obese, nondiabetic middle-aged Asian adults. Am J Gastroenterol 2012;107: 561-567.

26. Li Y, Xu C, Yu C, Xu L, Miao M. Association of serum uric acid level with non-alcoholic fatty liver disease: a cross-sectional study. J Hepatol 2009;50:1029-1034.

27. Lee JW, Cho YK, Ryan M, et al. Serum uric acid as a predictor for the development of nonalcoholic fatty liver disease in apparently healthy subjects: a 5-year retrospective cohort study. Gut Liver 2010;4:378-383.

28. Xu C, Yu C, Xu L, Miao M, Li Y. High serum uric acid increases the risk for nonalcoholic fatty liver disease: a prospective observational study. PLoS One 2010;5:e11578.

29. Hwang IC, Suh SY, Suh AR, Ahn HY. The relationship between normal serum uric acid and nonalcoholic fatty liver disease. J Korean Med Sci 2011;26:386-391.

30. Liu PW, Chang TY, Chen JD. Serum uric acid and metabolic syndrome in Taiwanese adults. Metabolism 2010;59:802-807.

31. Godsland IF, Lecamwasam K, Johnston DG. A systematic evaluation of the insulin resistance syndrome as an independent risk factor for cardiovascular disease mortality and derivation of a clinical index. Metabolism 2011;60:1442-1448.

32. Albano E, Mottaran E, Occhino G, Reale E, Vidali M. Review ar- ticle: role of oxidative stress in the progression of non-alcoholic steatosis. Aliment Pharmacol Ther 2005;22 Suppl 2:71-73.

33. Nieto FJ, Iribarren C, Gross MD, Comstock GW, Cutler RG. Uric acid and serum antioxidant capacity: a reaction to atherosclerosis? Atherosclerosis 2000;148:131-139.

34. Waring WS, Webb DJ, Maxwell SR. Systemic uric acid administration increases serum antioxidant capacity in healthy volunteers. J Cardiovasc Pharmacol 2001;38:365-371.

35. Garcia-Ruiz I, Rodriguez-Juan C, Diaz-Sanjuan T, et al. Uric acid and anti-TNF antibody improve mitochondrial dysfunction in ob/ ob mice. Hepatology 2006;44:581-591.

36. Hayden MR, Tyagi SC. Uric acid: a new look at an old risk marker for cardiovascular disease, metabolic syndrome, and type 2 diabetes mellitus: the urate redox shuttle. Nutr Metab (Lond) 2004;1:10.

37. Suzuki I, Yamauchi T, Onuma M, Nozaki S. Allopurinol, an inhibitor of uric acid synthesis: can it be used for the treatment of metabolic syndrome and related disorders? Drugs Today (Barc) 2009;45:363-378.

38. Sanchez-Lozada LG, Mu W, Roncal C, et al. Comparison of free fructose and glucose to sucrose in the ability to cause fatty liver. Eur J Nutr 2010;49:1-9.

39. Ratziu V, Giral P, Charlotte F, et al. Liver fibrosis in overweight patients. Gastroenterology 2000;118:1117-1123.

40. Sonsuz A, Basaranoglu M, Ozbay G. Relationship between aminotransferase levels and histopathological findings in patients with nonalcoholic steatohepatitis. Am J Gastroenterol 2000;95:13701371.

41. Hsiao TJ, Chen JC, Wang JD. Insulin resistance and ferritin as major determinants of nonalcoholic fatty liver disease in apparently healthy obese patients. Int J Obes Relat Metab Disord 2004;28: 167-172.

42. Zelber-Sagi S, Nitzan-Kaluski D, Halpern Z, Oren R. NAFLD and hyperinsulinemia are major determinants of serum ferritin levels. J Hepatol 2007;46:700-707.

43. Nelson JE, Wilson L, Brunt EM, et al. Relationship between the pattern of hepatic iron deposition and histological severity in nonalcoholic fatty liver disease. Hepatology 2011;53:448-457.

44. Imeryuz N, Tahan V, Sonsuz A, et al. Iron preloading aggravates nutritional steatohepatitis in rats by increasing apoptotic cell death. J Hepatol 2007;47:851-859.

45. Choi JS, Koh IU, Lee HJ, Kim WH, Song J. Effects of excess dietary iron and fat on glucose and lipid metabolism. J Nutr Biochem 2013;24:1634-1644.

46. Dongiovanni P, Ruscica M, Rametta R, et al. Dietary iron overload induces visceral adipose tissue insulin resistance. Am J Pathol 2013;182:2254-2263.

47. Choi ES, Kim MK, Song MK, et al. Association between serum irisin levels and non-alcoholic fatty liver disease in health screen examinees. PLoS One 2014;9:e110680. 